$01,11,19$

\title{
Изменение свойств железа при ОЦК-ГЦК-фазовом переходе
}

\author{
(C) М.Н. Магомедов \\ Институт проблем геотермии и возобновляемой энергетики - \\ филиал ФБГУН объединенного института высоких температур РАН, \\ Махачкала, Россия \\ E-mail: mahmag4@mail.ru
}

Поступила в Редакцию 30 сентября 2020 г.

В окончательной редакции 7 октября 2020 г.

Принята к публикации 8 октября 2020 г.

\begin{abstract}
Используя ранее разработанный метод расчета свойств кристалла на основе парного потенциала Ми-Леннард-Джонса, рассчитаны термодинамические свойства ОЦК- и ГЦК-фаз железа при температуре полиморфного ОЦК-ГЦК-фазового перехода. Рассчитаны 23 свойства железа и их изменения при ОЦК-ГЦК-переходе. Расчеты показали, что такие свойства как: параметр Грюнайзена, коэффициент теплового расширения и теплоемкость практически не изменяются при ОЦК-ГЦК-переходе. Модуль упругости, удельная энтропия, коэффициент Пуассона и удельная поверхностная энергия изменяются так же, как и молярный объем, т. е. в пределах $1 \%$. Температура Дебая и ее производная по давлению уменьшаются при ОЦК-ГЦК-переходе так же, как увеличивается расстояние между центрами ближайших атомов, т. е. в пределах 2-3\%. На основе анализа известных из литературы экспериментальных данных показано, что даже сравнительно точно измеримые параметры как коэффициент теплового расширения и модуль упругости измеряются с погрешностью превышающей значения скачков этих параметров при ОЦК-ГЦК-переходе. Указано, что аморфизация либо наноструктурирование определенной доли железа в процессе ОЦК-ГЦКперехода может вносить вклад в изменения свойств железа при данном фазовом переходе.
\end{abstract}

Ключевые слова: железо, межатомный потенциал, ОЦК- и ГЦК-структура, полиморфный переход.

DOI: 10.21883/FTT.2021.02.50462.209

\section{1. Введение}

Как известно из экспериментов [1-7] железо (Fe) при низких давлениях $(P \cong 0)$ и при нагреве выше температуры $T_{f}=1184 \mathrm{~K}$ переходит из объемноцентрированной кубической $($ ОЦК, $\operatorname{Im} 3 \mathrm{~m}) \quad \alpha$-фазы (феррит) в гранецентрированную кубическую (ГЦК, Fm3m) $\gamma$-фазу (аустенит). Данный полиморфный $\alpha \rightarrow \gamma$-переход является фазовым переходом первого рода. Поэтому при этом переходе молярный объем железа изменяется, уменьшаясь [5] от $V(\alpha)=7.37$ до $V(\gamma)=7.30\left[\mathrm{~cm}^{3} / \mathrm{mol}\right]$. Таким образом, изменение объема при этом переходе равно: $\Delta V=V(\gamma)-V(\alpha)=-0.074[1] ;-0.07[5]\left[\mathrm{cm}^{3} / \mathrm{mol}\right], \mathrm{a}$ относительный скачек объема равен

$$
\begin{aligned}
\Delta V / V(\gamma) & =[V(\gamma)-V(\alpha)] / V(\alpha)=-0.01[1] ; \\
& -0.0095[5] ; \quad-0.0096[7] \cong-1 \% .
\end{aligned}
$$

Молярная энтропия при этом переходе возрастает на величину [1]: $\Delta S=S(\gamma)-S(\alpha)=0.69 \mathrm{~J} /(\mathrm{K} \cdot \mathrm{mol})$, т. е. удельная (на атом) нормированная энтропия возрастает на величину $\Delta s / k_{B}=0.083$, где $k_{B}$ - постоянная Больцмана.

Параметр решетки для ОЦК- и ГЦК-фаз железа при $P \cong 0$ и $T_{f}=1184 \mathrm{~K}$ равен [7]:

$$
l(\alpha-\mathrm{Fe})=2.867 \quad \text { и } \quad l(\gamma-\mathrm{Fe})=3.651\left[10^{-10} \mathrm{~m}\right] .
$$

Так как расстояние между центрами ближайших атомов связано с параметром решетки соотношением: $c($ ОЦК $)=\left(3^{1 / 2} / 2\right) l=0.866 l$ и $c($ ГЦК $)=\left(1 / 2^{1 / 2}\right) l$ $=0.7071 l$, то из этих формул и данных представленных в [7] получим

$$
c(\alpha-\mathrm{Fe})=2.4828 \quad \text { и } \quad c(\gamma-\mathrm{Fe})=2.5816\left[10^{10} \mathrm{~m}\right],
$$

т. е. $\Delta c / c(\alpha)=[c(\gamma)-c(\alpha)] / c(\alpha)=0.0398$.

Несмотря на то что $\alpha \rightarrow \gamma$-переход изучается давно, но изменение термодинамических свойств при этом переходе изучены не достаточно точно. Это связано с экспериментальными трудностями, обусловленными тем, что при температуре Кюри $T_{C}=1043 \mathrm{~K}$ в железе происходит фазовый переход второго рода из ферромагнитного в парамагнитное состояние. Так как $T_{C}$ всего на $141 \mathrm{~K}$ ниже чем $T_{f}$, то это соседство затрудняет измерение различных термодинамических свойств при температуре $\alpha \rightarrow \gamma$-перехода, что и отмечалось в работах [1-5].

Более того, в связи с тем, что при $\alpha \rightarrow \gamma$-переходе происходит такое малое изменение объема и связанных с ним других свойств, то этот скачек свойств очень трудно экспериментально изменить. Дело усугубляется как близостью температуры Кюри, так и тем, что погрешность измерения некоторых свойств много больше величины скачка этих свойств при $\alpha \rightarrow \gamma$-переходе. Например, в [8, Fig. 5] показано, что изменение модуля упругости $\left(B_{T}=-V(\partial P / \partial V)_{T}\right)$ при $\alpha \rightarrow \gamma$-переходе меньше, чем 
величина погрешности экспериментального определения величины $B_{T}$.

Изучение $\alpha \rightarrow \gamma$-перехода в железе методами компьютерного моделирования не позволили получить не только величину изменения свойства, но даже не позволили однозначно ответить о знаке изменения свойства при этом переходе [7-9]. В связи с этим, в настоящей работе были рассчитаны свойства $\alpha$ - и $\gamma$-фаз железа при $P=0$ и $T_{f}=1184 \mathrm{~K}$ аналитическим методом, изложенным в работах [10-12]. Цель этой работы - на основе единого метода рассчитать изменение всех термодинамических параметров железа при $\alpha \rightarrow \gamma$-переходе.

\section{2. Метод расчета свойств однокомпонентного кристалла}

Для расчета решеточных свойств кристалла однокомпонентного вещества необходимо определить, как потенциал взаимодействия пары его атомов, так и метод расчета, который основан на этом потенциале. Представим парное межатомное взаимодействие в виде потенциала Ми-Леннард-Джонса, который имеет вид [13]:

$$
\varphi(r)=\frac{D}{(b-a)}\left[a\left(\frac{r_{0}}{r}\right)^{b}-b\left(\frac{r_{0}}{r}\right)^{a}\right]
$$

где $D$ и $r_{0}$ - глубина и координата минимума потенциала, $b>a>1$ - численные параметры.

Тогда, используя приближение „взаимодействия только ближайших соседей“ температуру Дебая как функцию первого координационного числа $\left(k_{n}\right)$ и расстояния между центрами ближайших атомов можно определить из выражения [14]:

$$
\Theta\left(k_{n}, c\right)=A_{w}\left(k_{n}, c\right) \xi\left[-1+\left(1+\frac{8 D}{k_{B} A_{w}\left(k_{n}, c\right) \xi^{2}}\right)^{1 / 2}\right] .
$$

Здесь $c=\left(6 k_{p} v / \pi\right)^{1 / 3}-$ расстояние между центрами ближайших атомов, $v=V / N-$ удельный объем, $V$ и $N$ - объем и число атомов в кристалле, $k_{p}-$ коэффициент упаковки.

Функция $A_{w}$ возникает в (2) из-за учета энергии „нулевых колебанийс атомов в кристалле

$$
\begin{gathered}
A_{w}\left(k_{n}, c\right)=K_{R} \frac{5 k_{n} a b(b+1)}{144(b-a)}\left(\frac{r_{0}}{c}\right)^{b+2}, \\
K_{R}=\frac{\hbar^{2}}{k_{B} r_{0}^{2} m}, \quad \xi=\frac{9}{k_{n}},
\end{gathered}
$$

где $m$ - масса атома, $\hbar-$ постоянная Планка, $k_{n}-$ первое координационное число.

Используя для колебательного спектра кристалла модель Эйнштейна, для удельной (на атом) свободной энергии Гельмгольца кристалла можно принять выражение [10-12,15]:

$$
\begin{aligned}
& f_{H}\left(k_{n}, c, T\right)=\left(\frac{k_{n}}{2} D\right) U(R)+3 k_{B} \Theta_{E}\left(k_{n}, c\right) \\
& \times\left\{\frac{1}{2}+\left(\frac{T}{\Theta_{E}\left(k_{n}, c\right)}\right) \ln \left[1-\exp \left(-\frac{\Theta_{E}\left(k_{n}, c\right)}{T}\right)\right]\right\},
\end{aligned}
$$

где $T$ - температура, $\Theta_{E}-$ температура Эйнштейна, которая связана с температурой Дебая соотношением [15]: $\Theta=(4 / 3) \Theta_{E}, R=r_{0} / c-$ относительная линейная плотность кристалла, функция потенциальной энергии, в соответствии с (1), равна

$$
U(R)=\frac{a R^{b}-b R^{a}}{b-a} .
$$

Тогда из (4) для уравнения состояния $P\left(k_{n}, v, T\right)$ и изотермического модуля упругости $\left(B_{T}\right)$ можно получить [10-12]:

$$
P=-\left(\frac{\partial f_{H}}{\partial v}\right)_{T}=\left[\frac{k_{n}}{6} D U^{\prime}(R)+3 k_{B} \Theta_{E} \gamma E_{w}\left(\frac{\Theta_{E}}{T}\right)\right] \frac{1}{v},
$$

$$
\begin{aligned}
B_{T}= & -v\left(\frac{\partial P}{\partial v}\right)_{T} \\
= & P+\left[\frac{k_{n}}{18} D U^{\prime \prime}(R)+3 k_{B} \Theta_{E} \gamma(\gamma-q) E_{w}\left(\frac{\Theta_{E}}{T}\right)\right. \\
& \left.-3 N k_{B} \gamma^{2} T F_{E}\left(\frac{\Theta_{E}}{T}\right)\right] \frac{1}{v} .
\end{aligned}
$$

Здесь введены следующие функции:

$$
\begin{gathered}
E_{w}(y)=0.5+\frac{1}{[\exp (y)-1]}, \quad F_{E}(y)=\frac{y^{2} \exp (y)}{[\exp (y)-1]^{2}}, \\
U^{\prime}(R)=R\left[\frac{\partial U(R)}{\partial R}\right]=\frac{a b\left(R^{b}-R^{a}\right)}{b-a}, \\
U^{\prime \prime}(R)=R\left[\frac{\partial U^{\prime}(R)}{\partial R}\right]=\frac{a b\left(b R^{b}-a R^{a}\right)}{b-a} .
\end{gathered}
$$

Из формулы (2) легко найти выражения для первого $(\gamma)$, второго $(q)$ и третьего $(z)$ параметров Грюнайзена

$$
\begin{gathered}
\gamma=-\left(\frac{\partial \ln \Theta}{\partial \ln v}\right)_{T}=\frac{b+2}{6\left(1+X_{w}\right)}, \\
q=\left(\frac{\partial \ln \gamma}{\partial \ln v}\right)_{T}=\gamma \frac{X_{w}\left(1+2 X_{w}\right)}{\left(1+X_{w}\right)} \\
z=-\left(\frac{\partial \ln q}{\partial \ln v}\right)_{T}=\gamma\left(1+4 X_{w}\right)-2 q=\gamma\left(\frac{1+3 X_{w}}{1+X_{w}}\right) \\
=\frac{(b+2)}{6} \frac{\left(1+3 X_{w}\right)}{\left(1+X_{w}\right)^{2}} .
\end{gathered}
$$

где введена функция: $X_{w}=A_{w} \xi / \Theta$, которая определяет роль квантовых эффектов. 
Так как температура Дебая (2) не зависит от температуры при изохорическом нагреве, то изохорную и изобарную теплоемкости можно определить в следующем виде $[10-12,15]$ :

$$
C_{v}=3 N k_{B} F_{E}\left(\frac{\Theta_{E}}{T}\right), \quad C_{p}=C_{v}\left(1+\gamma \alpha_{p} T\right),
$$

где изобарный коэффициент теплового объемного расширения можно рассчитать по уравнению Грюнайзена [15]:

$$
\alpha_{p}=\frac{\gamma C_{v}}{V B_{T}}=\frac{\gamma C_{v}}{N B_{T}\left[\pi r_{0}^{3} /\left(6 k_{p}\right)\right]}\left(\frac{\nu_{0}}{v}\right), \quad v_{0}=\frac{\pi r_{0}^{3}}{6 k_{p}} .
$$

Из формулы (4) также можно рассчитать значение удельной (на атом) энтропии системы $[12,15]$ :

$$
\begin{aligned}
s & =-\left(\frac{\partial f_{H}}{\partial T}\right)_{v} \\
& =3 k_{B}\left\{-\ln \left[1-\exp \left(\frac{\Theta_{E}}{T}\right)\right]+\left(\frac{\Theta_{E}}{T}\right) /\left[\exp \left(\frac{\Theta_{E}}{T}\right)-1\right]\right\}
\end{aligned}
$$

B рамках RP-модели из [12] для удельной (на единицу площади) поверхностной энергии грани (100) кристалла $(\sigma)$, ее изохорной и изобарной производных по температуре можно получить следующие выражения:

$$
\begin{gathered}
\sigma=-\frac{k_{n} D R^{2}}{12 \alpha^{2 / 3} r_{0}^{2}}\left[U(R)+\frac{18 \gamma}{(b+2)} \frac{k_{B} \Theta_{E}}{D k_{n}} E_{w}\left(\frac{\Theta_{E}}{T}\right)\right], \\
\sigma^{\prime}(T)_{v}=\left(\frac{\partial \sigma}{\partial T}\right)_{v}=-\frac{3 k_{B} R^{2} \gamma}{2 \alpha^{2 / 3}(b+2) r_{0}^{2}} F_{E}\left(\frac{\Theta_{E}}{T}\right), \\
\sigma^{\prime}(T)_{p}=\left(\frac{\partial \sigma}{\partial T}\right)_{P}=\sigma^{\prime}(T)_{v}+v \alpha_{p}\left(\frac{\partial \sigma}{\partial v}\right)_{T} \\
=\sigma^{\prime}(T)_{v}-\frac{2}{3} \sigma \alpha_{p} \Delta_{p} .
\end{gathered}
$$

Здесь введенные функции имеют следующий вид:

$$
\begin{gathered}
\Delta_{p}=-\frac{1}{2}\left[\frac{\partial \ln (\sigma)}{\partial \ln (c)}\right]_{T}=1+\left\{U^{\prime}(R)-\left[q-\gamma t_{y}\left(\frac{\Theta_{E}}{T}\right)\right]\right. \\
\left.\times \frac{54 \gamma}{(b+2)} \frac{k_{B} \Theta_{E}}{D k_{n}} E_{w}\left(\frac{\Theta_{E}}{T}\right)\right\} / \\
\left\{2\left[U(R)+\frac{18 \gamma}{(b+2)} \frac{k_{B} \Theta_{E}}{D k_{n}} E_{w}\left(\frac{\Theta_{E}}{T}\right)\right]\right\}, \\
t_{y}(y)=1-\frac{2 y \exp (y)}{[\exp (2 y)-1]} .
\end{gathered}
$$

При $T \rightarrow 0 \mathrm{~K}$ функции из (13) и (14) стремятся к нулю при любом $R$, что согласуется с третьим началом термодинамики в „сильной“ формулировке Планка.
Коэффициент Пуассона $\mu_{p}$ кристалла при различных $R-T$-условиях можно рассчитать из соотношения [16]:

$$
\mu_{p}=\frac{1}{2}-\frac{1}{48 X_{s c} \gamma^{2}}, \quad X_{s c}=\frac{\sigma}{c B_{T}} .
$$

Полученные выражения (2)-(15) позволяют рассчитать зависимость, как уравнения состояния, так и указанных свойств от нормированного объема $v / \nu_{0}=\left(c / r_{0}\right)^{3}=R^{-3}$ и температуры $T$ для однокомпонентного кристалла с заданной структурой (т.е. при данных значениях $k_{n}$ и $k_{p}$ ) если известны параметры межатомного потенциала (1).

\section{3. Определение параметров межатомного потенциала}

Применим формализм (2)-(15) для расчета свойств железа (Fe, масса атома $m=55.847$ a.m.u.) с различной структурой. Для ОЦК- $\alpha$ - $\mathrm{Fe}$ (где $k_{n}=8$ и $k_{p}=0.6802$ ) параметры потенциала (1) были определены самосогласованным методом в работах $[10,13]$, и они имеют следующий вид:

$$
\begin{gathered}
r_{0}(\alpha)=2.4775 \cdot 10^{-10} \mathrm{~m}, \quad D(\alpha) / k_{B}=12561.53 \mathrm{~K}, \\
a(\alpha)=3.09, \quad b(\alpha)=8.37 .
\end{gathered}
$$

Что касается высокотемпературной ГЦК- $\gamma$-фазы железа (где $k_{n}=12$ и $k_{p}=0.7405$ ), то если использовать для нее параметры потенциала (16), то при $P=0$ и $T_{f}=1184 \mathrm{~K}$ с помощью формализма из $(2)-(15)$ получим для скачка объема: $\Delta V / V(\alpha)=-9.63 \%$, что на порядок больше экспериментальной величины. Это связано с тем, что при изменении структуры кристалла происходит перераспределение электронной плотности, что и приводит к изменению параметров парного межатомного потенциала.

Поэтому для ГЦК- $\gamma$-фазы железа параметры потенциала (1) были получены из параметров $r_{0}(\alpha)$ и $D(\alpha)$ потенциала (16) для $\alpha$-Fe-методом, который был использован в работах $[11,17]$. Суть метода в следующем. Если при полиморфном превращении величина первого координационного числа $k_{n}$ возрастает от 8 до 12 , то расстояние между центрами ближайших атомов возрастает в соотношении [18, p. 288]: $r_{0}(12)=(1.14 / 1.11) r_{0}(8)$. Так как при переходе от ОЦК- к ГЦК-структуре значение энергии межатомной связи кристалла перераспределяется от $k_{n}=8$ связей с ближайшими соседями к связям с $k_{n}=12$, то величина глубины парной межатомной связи для ГЦК-кристалла можно оценить по формуле: $D(12)=(8 / 12) D(8)$. При этом мы полагаем, что степенные параметры потенциала (1) $a$ и $b$ при полиморфном превращении не изменяются. Несмотря на свою простоту, данный метод позволил получить параметры потенциала, из которых были рассчитаны свойства различных фаз $\mathrm{Fe}$ [11], Si и $\mathrm{Ge}$ [17]. 
Таблица 1. Рассчитанные свойства $\alpha$-Fе и $\gamma$-Fe железа при $P=0$ и $T_{f}=1184 \mathrm{~K}$

\begin{tabular}{|c|c|c|c|}
\hline hfil Свойство [единица измерения] & $\alpha$-Fe & $\gamma$-Fe & $\Delta X \times 100$ \\
\hline $\begin{array}{l}V=\left[\pi N /\left(6 k_{p}\right)\right] c^{3}\left[\mathrm{~cm}^{3} / \mathrm{mol}\right] \\
c\left[10^{-10} \mathrm{~m}\right] \\
\Theta-\text { температура Дебая }[\mathrm{K}] \\
\gamma=-(\partial \ln \theta / \partial \ln V)_{T} \\
q \cdot 10^{3}=\left[(\partial \ln \gamma / \partial \ln V)_{T}\right] \cdot 10^{3} \\
z=-(\partial \ln q / \partial \ln V)_{T} \\
B_{T}=-V(\partial P / \partial V)_{T}[\mathrm{GPa}] \\
B^{\prime}(P)=\left(\partial B_{T} / \partial P\right)_{T}^{1)} \\
\alpha_{p}=\gamma C_{v} /\left(B{ }_{T} V\right)\left[10^{-6} \mathrm{~K}^{-1}\right] \\
\alpha_{p} B_{T}=(\partial P / \partial T)_{V}=(\partial S / \partial V)_{T}\left[10^{-3} \mathrm{GPa} / \mathrm{K}\right] \\
C_{v} /\left(N k_{B}\right)-\text { изохорная теплоемкость } \\
C_{p} /\left(N k_{B}\right)=\left[C_{v} /\left(N k_{B}\right)\right]\left(1+\gamma \alpha_{p} T\right) \\
S /\left(N k_{B}\right)=s / k_{B}-\text { нормированная энтропия } \\
\Theta^{\prime}(P)=(\partial \Theta / \partial P)_{T}[\mathrm{~K} / \mathrm{GPa}]^{1)} \\
C_{v}^{\prime}(P) /\left(N k_{B}\right)\left[10^{-3} / \mathrm{GPa}\right]^{1)} \\
\alpha_{p}^{\prime}(P)=\left(\partial \alpha_{p} / \partial P\right)_{T}\left[10^{-6} /(\mathrm{K} \cdot \mathrm{GPa})\right]^{1)} \\
C_{p}^{\prime}(P) /\left(N k_{B}\right)\left[10^{-3} / \mathrm{GPa}\right]^{1)} \\
\sigma(100)\left[10^{-3} \mathrm{~J} / \mathrm{m}^{2}\right] \\
X_{s c} \cdot 10^{3}=10^{3} \cdot \sigma(100) /\left(c B_{T}\right) \\
\mu_{p}-\text { коэффициент Пуассона } \\
\sigma^{\prime}(T)_{v}=(\partial \sigma / \partial T)_{v}\left[10^{-6} \mathrm{~J} /\left(\mathrm{m}^{2} \mathrm{~K}\right)\right] \\
\sigma^{\prime}(T)_{P}=(\partial \sigma / \partial T)_{P}\left[10^{-6} \mathrm{~J} /\left(\mathrm{m}^{2} \mathrm{~K}\right)\right] \\
\sigma^{\prime}(P)_{T}=(\partial \sigma / \partial P)_{T}\left[10^{-3} \mathrm{~J} /\left(\mathrm{m}^{2} \mathrm{GPa}\right)\right]^{1)}\end{array}$ & $\begin{array}{r}7.3847 \\
2.5162 \\
391.19 \\
1.7207 \\
7.6357 \\
1.7359 \\
135.73 \\
5.9665 \\
42.602 \\
5.7823 \\
2.9847 \\
3.2437 \\
7.1930 \\
4.9567 \\
-0.3864 \\
-1.5661 \\
-9.9569 \\
2091.0 \\
61.227 \\
0.3851 \\
-64.294 \\
-134.98 \\
12.220\end{array}$ & $\begin{array}{r}7.3135 \\
2.5801 \\
381.55 \\
1.7209 \\
7.4459 \\
1.7357 \\
137.05 \\
5.9668 \\
42.619 \\
5.8409 \\
2.9854 \\
3.2447 \\
7.2676 \\
4.7885 \\
-0.3827 \\
-1.5517 \\
-9.8684 \\
2104.6 \\
59.521 \\
0.3818 \\
-64.736 \\
-135.91 \\
12.183\end{array}$ & $\begin{array}{r}-0.964 \\
2.539 \\
-2.465 \\
0.013 \\
-2.486 \\
-0.013 \\
0.972 \\
0.004 \\
0.040 \\
1.014 \\
0.025 \\
0.031 \\
1.037 \\
-3.394 \\
0.949 \\
0.919 \\
0.889 \\
0.651 \\
-2.787 \\
-0.854 \\
-0.687 \\
-0.690 \\
-0.306\end{array}$ \\
\hline
\end{tabular}

Примечание. 1) Рассчитано численным дифференцированием параметра по давлению вдоль изотермы.

Таким методом для ГЦК- $-\mathrm{Fe}$ были получены следующие значения параметров потенциала (1): $r_{0}(\gamma)=2.5445 \cdot 10^{-10} \mathrm{~m}, D(\gamma) / k_{B}=8374.353 \mathrm{~K}, a(\gamma)=$ $=3.09, b(\gamma)=8.37$.

Но при расчетах свойств ГЦК- $\gamma$-Fе-методом из [10-12] эти параметры показали плохие результаты для скачка объема: $\Delta V / V(\alpha)=-0.488 \%$, что в два раза меньше экспериментальной величины. Поэтому в последующем была сделана подгонка величины $r_{0}(\gamma)$ до получения экспериментального значения [7]: $\Delta V / V(\alpha)=-0.96 \%$. Таким образом, для ГЦК- $\gamma$-Fe было получено

$$
\begin{gathered}
r_{0}(\gamma)=2.5404 \cdot 10^{-10} \mathrm{~m}, \quad D(\gamma) / k_{B}=8374.353 \mathrm{~K}, \\
a(\gamma)=3.09, \quad b(\gamma)=8.37 .
\end{gathered}
$$

Отметим, что параметры (17) мало отличаются от параметров потенциала (1), которые были получены для ГЦК- $\gamma$-Fе в работе [19] иным методом расчета:

$$
\begin{gathered}
r_{0}(\gamma)=2.517 \cdot 10^{-10} \mathrm{~m}, \quad D(\gamma) / k_{B}=8130.36 \mathrm{~K}, \\
a(\gamma)=3.5, b(\gamma)=7.0 .
\end{gathered}
$$

Но при расчетах свойств ГЦК- $\gamma$-Fе-методом из [10-12] параметры (18) показали худшие результаты для $\Delta V / V(\alpha)$, чем параметры (17). Поэтому при расчете свойств $\alpha$ - и $\gamma$-фаз Fе при $T_{f}=1184 \mathrm{~K}$ мы использовали параметры (16) и (17) соответственно.

\section{4. Результаты расчета свойств железа и их обсуждение}

В табл. 1 представлены рассчитанные с помощью формализма из $(2)-(15)$ при $P=0$ и $T_{f}=1184 \mathrm{~K}$ свойства (они указаны в первом столбце) для $\alpha$ - и $\gamma$-фаз железа. Во втором и третьем столбцах табл. 1 представлены абсолютные значения свойств для $\alpha$-Fe и $\gamma$-Fe, а в четвертом столбце представлена величина их относительного изменения (в \%): $\Delta X=[X(\gamma)-X(\alpha)] / X(\alpha)$.

Из представленных в табл. 1 результатов можно сделать следующие выводы:

1. Рассчитанные нами молярные объемы получились больше, чем экспериментальные значения [5]: $V(\alpha)=7.37$ и $\left.V(\gamma)=7.30 \mathrm{~cm}^{3} / \mathrm{mol}\right]$. Изменение молярного объема у нас составляет: $\Delta V=-0.0712\left[\mathrm{~cm}^{3} / \mathrm{mol}\right]$, что сравнимо с экспериментальной величиной в пределах точности ее определения.

2. Расчеты расстояния между центрами ближайших атомов показали: для ОЦК-фазы рассчитанная $c(\alpha$-Fe $)$ больше, чем экспериментальная величина [7]: $c(\alpha-\mathrm{Fe})=2.4828$; для ГЦК-фазы рассчитанная величина сравнима с экспериментальным значением [7]: $c(\gamma$-Fe $)=2.5816\left[10^{-10} \mathrm{~m}\right]$. Поэтому скачок $\Delta c / c(\alpha)$ получился у нас чуть меньше экспериментального значения [7]: $\Delta c / c(\alpha)=3.98 \%$.

3. Расчет изменения удельной энтропии при $\alpha \rightarrow \gamma$ переходе показал: $\Delta s / k_{B}=0.0746$. Это согласуется с 
Таблица 2. Экспериментальные и теоретические (из [8]) оценки коэффициента теплового расширения железа при $\alpha \rightarrow \gamma$ переходе. Здесь $\Delta \alpha_{p}=\left[\alpha_{p}(\gamma)-\alpha_{p}(\alpha)\right] / \alpha_{p}(\alpha)$

\begin{tabular}{l|c|c|c}
\hline Источник данных & $\alpha_{p}(\mathrm{bcc}-\mathrm{Fe}), 10^{-6} \mathrm{~K}^{-1}$ & $\alpha_{p}(\mathrm{fcc}-\mathrm{Fe}), 10^{-6} \mathrm{~K}^{-1}$ & $\Delta \alpha_{p}, \%$ \\
\hline Basinski-1955 [24] & 43.8 & 70.8 & 61.6 \\
Novikova-1974 [2] & 46.5 & 67.2 & 44.5 \\
Acet-1994 [25] & 42.0 & 67.2 & 60.0 \\
Liu-2004 [4] & $51.4 \pm 3$ & $69.6 \pm 3$ & 35.4 \\
Balagurov-2018 [7] & 50.0 & 55.0 & 10.0 \\
\hline Theory: Dong-2017 [8] & & & \\
LSF scheme & 49.5 & 81.6 & 64.8 \\
FS scheme & 47.4 & 85.8 & 81.0
\end{tabular}

экспериментальной оценкой $[1]: \Delta s / k_{B}=0.083$, в пределах точности ее определения.

4. Из наших результатов наклон линии $\alpha \rightarrow \gamma$-перехода при $P=0$ получился равным: $\partial T_{f} / \partial P=\Delta V / \Delta S=-114.8 \mathrm{~K} / \mathrm{GPa}$. Это хорошо согласуется как с оценкой из обзора [1]: $\partial T_{f} / \partial P=$ $=-105 \pm 5 \mathrm{~K} / \mathrm{GPa}$, так и с более свежими данными из работ $[20,21]$.

5. Температура Дебая при $\alpha \rightarrow \gamma$-переходе в железе уменьшается. Это отмечалось и в работах других авторов: $\Theta(\alpha)=419 \mathrm{~K}$ и $\Theta(\gamma)=335 \mathrm{~K}[22] ; \Theta(\alpha)=303 \mathrm{~K}$ и $\Theta(\gamma)=225.5 \mathrm{~K}$ [23]. Отметим, что расчеты $\alpha \rightarrow \gamma$ перехода из [8, Fig. 5] показали неизменность $\Theta$ при расчете по FS-схеме и увеличение $\Theta$ при расчете по LSF-схеме.

6. Первый параметр Грюнайзена $\gamma$ при $\alpha \rightarrow \gamma$-переходе в железе незначительно увеличивается, что согласуется с результатами работы [6].

7. Второй $(q)$ и третий $(z)$ параметры Грюнайзена при $\alpha \rightarrow \gamma$-переходе в железе уменьшаются.

8. Модуль упругости $B_{T}$ при $\alpha \rightarrow \gamma$-переходе в железе увеличивается, что согласуется с результатами работ $[6,23]$. Отметим, что расчеты из $[8$, Fig. 5] показали уменышение величины $B_{T}$ при $\alpha \rightarrow \gamma$-переходе.

9. Производная функции $B_{T}$ по давлению при $\alpha \rightarrow \gamma$ переходе в железе при $P=0$ незначительно уменьшается, что согласуется с результатами работы [23].

10. Коэффициент теплового расширения $\alpha_{p}$ при $\alpha \rightarrow \gamma$-переходе в железе незначительно увеличивается. Увеличение $\alpha_{p}$ при $\alpha \rightarrow \gamma$-переходе отмечалось в работах [2,4,7,8,23-25]. Эти данные представлены в табл. 2. При этом, как это отмечалось в работах [2, стр. 199; 4], близость температуры Кюри приводит к различию экспериментальных данных для $\alpha_{p}(\alpha$-Fe $)$ в 1.5 раза. Поэтому, как видно из табл. 2 , величина $\Delta \alpha_{p}=\left[\alpha_{p}(\gamma)-\alpha_{p}(\alpha)\right] / \alpha_{p}(\alpha)$ изменяется в очень широких пределах.

11. Значения произведения $\alpha_{p} \cdot B_{T}$ и молярных теплоемкостей $C_{v}$ и $C_{p}$ при температуре $\alpha \rightarrow \gamma$-перехода в железе незначительно увеличиваются.

12. Производная температуры Дебая по давлению уменьшается при $\alpha \rightarrow \gamma$-переходе. Это согласуется с результатами работы [22]: $\Theta^{\prime}(P)_{\alpha}=4.89 \mathrm{~K} / \mathrm{GPa}$ и $\Theta^{\prime}(P)_{\gamma}=1.38 \mathrm{~K} / \mathrm{GPa}$.

13. Изотермические производные $\alpha_{p}$ и молярных теплоемкостей $\left(C_{v}\right.$ и $\left.C_{p}\right)$ по давлению возрастают при $\alpha \rightarrow \gamma$-переходе.

14. Удельная поверхностная энергия грани (100) железа возрастает при $\alpha \rightarrow \gamma$-переходе. Это отмечалось и в работах других авторов: $\sigma(\alpha)=1910$ и $\sigma(\gamma)=2170 \cdot 10^{-3} \mathrm{~J} / \mathrm{m}^{2} \quad[26] ; \quad \sigma(\alpha)=2040 \quad$ и $\sigma(\gamma)=2170 \cdot 10^{-3} \mathrm{~J} / \mathrm{m}^{2} \quad[27] ; \quad \sigma(\alpha)=2482$ и $\sigma(\gamma)=$ $=3372 \cdot 10^{-3} \mathrm{~J} / \mathrm{m}^{2}$ [28]. Отметим, что из литературы известны теоретические результаты с противоположным соотношением величины $\sigma(100)$ для ОЦК- и ГЦК-фаз железа (например [29]). Но результаты эти получены, как правило, при $T=0 \mathrm{~K}$ и при неопределенном значении давления.

15. Коэффициент Пуассона уменьшается при $\alpha \rightarrow \gamma$ переходе. Это согласуется с результатами, представленными в работах [30;32, стр. 313].

16. Производные удельной поверхностной энергии грани (100) по температуре и по давлению уменьшаются при $\alpha \rightarrow \gamma$-переходе в железе при $P=0$ и $T_{f}=1184 \mathrm{~K}$.

Отметим, что при ОЦК $\rightarrow$ ГЦК-переходе происходят два противоположных процесса: с одной стороны уплотнение структуры, а с другой стороны - увеличение расстояния между центрами ближайших атомов. Поэтому при $\alpha \rightarrow \gamma$-переходе происходят столь малые изменения свойств, что их очень трудно экспериментально измерить. Даже один из самых точно измеряемых параметров - коэффициент теплового расширения определяется при $\alpha \rightarrow \gamma$-переходе с большой погрешностью $[2,4]$.

На рисунке показана зависимость давления (в $\mathrm{GPa}$ ) от молярного объема (в $\mathrm{cm}^{3} / \mathrm{mol}$ ) в области $\alpha-\gamma$ перехода. Сплошные линии на рисунке - расчеты по формуле (5) при $T_{f}=1184 \mathrm{~K}$ : верхняя кривая для ОЦК-Fe, нижняя - для ГЦК-Fe. Треугольники значения объема при $P=0$ и $T_{f}=1184 \mathrm{~K}$ из $[1,3,5]$ : сплошной треугольник - объем ГЦК-Fе, открытый треугольник - объем ОЦК-Fе. Сплошные кружки данные из [32] для ГЦК-Fе, полученные в области 


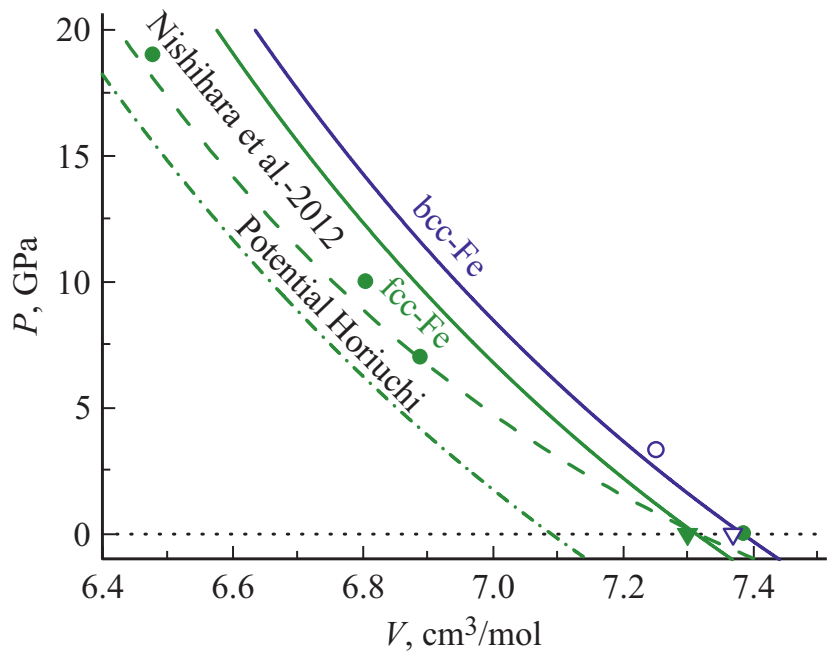

Зависимость давления от молярного объема в области $\alpha \rightarrow \gamma$ перехода в железе. Сплошные линии - расчеты по формуле (5) при $T_{f}=1184 \mathrm{~K}$ : верхняя кривая - для ОЦК-Fе, нижняя - для ГЦК-Fе. Треугольники - значения объема при $P=0$ и $T_{f}=1184 \mathrm{~K}$ из $[1,3,5]:$ сплошной треугольник - объем ГЦК-Fе, открытый треугольник - объем ОЦК-Fe. Сплошные кружки - данные из [32] для ГЦК-Fе, полученные в области температур 1000-1150 К. Открытый кружок - данные из [32] для ОЦК-Fe, полученные при температуре $1020 \mathrm{~K}$. Штрихпунктирная линия - расчет уравнения состояния ГЦК-Fе с использованием параметров потенциала (18) из работы Horiuchi et al. [19]. Штриховая линия - аппроксимация экспериментальных данных, полученных в работе Nishihara et al. [33] при $1073 \mathrm{~K}$ для ГЦК-Fe, уравнением состояния BirchMurnaghan (см. текст).

температур 1000-1150 K. Открытый кружок - данные из [32] для ОЦК-Fе, полученные при температуре 1020 К. Штрих-пунктирная линия — расчет уравнения состояния ГЦК-Fе с использованием параметров потенциала (18) из работы Horiuchi T. et al. [19].

На рисунке штриховой линией показана аппроксимация экспериментальных данных, полученных в работе Nishihara et al. [33] при $1073 \mathrm{~K}$ для ГЦК-Fe, уравнением состояния Birch-Murnaghan 3rd ordered, которое имеет вид:

$$
\begin{aligned}
P= & \frac{3}{2} B_{0 T}\left[\left({\frac{V_{0 T}}{V}}^{7 / 3}-\left(\frac{V_{0 T}}{V}\right)^{5 / 3}\right]\right. \\
& \times\left\{1+\frac{3}{4}\left(B_{0 T}^{\prime}-4\right)\left[\left(\frac{V_{0 T}}{V}\right)^{2 / 3}-1\right]\right\} .
\end{aligned}
$$

Здесь $V_{0 T}, B_{0 T}, B_{0 T}^{\prime}$ - это подгоночные параметры, которые для ГЦК-Fe, согласно [33], равны

$$
\begin{gathered}
V_{0 T}=48.600 \pm 0.098 \AA^{3} / \text { at }=7.3169 \pm 0.0148 \mathrm{~cm}^{3} / \mathrm{mol}, \\
B_{0 T}=88.9 \pm 5.1 \mathrm{GPa}, \quad B_{0 T}^{\prime}=8.9 \pm 0.7
\end{gathered}
$$

Таким образом, из данных работы [33] можно оценить точность экспериментального определения указанных параметров: $[X]= \pm \delta X / X$. Из (19) получим

$$
\left[V_{0 T}\right]= \pm 0.02 \%, \quad\left[B_{0 T}\right]= \pm 5.7 \%, \quad\left[B_{0 T}^{\prime}\right]= \pm 7.9 \%
$$

Из этих данных (табл. 1 и рисунок) можно легко понять, что даже при $P=0$ погрешность определения упругих параметров $B_{T}$ и $B^{\prime}(P)$ при $\alpha \rightarrow \gamma$-переходе больше, чем значения скачков этих параметров при этом фазовом переходе.

В работе [7] было обнаружено, что в процессе $\alpha \rightarrow \gamma$ перехода в интервале температур от $1183 \mathrm{~K}$ до $1208 \mathrm{~K}$ образуется до 10-20\% аморфного состояния железа. Появившись при $1183 \mathrm{~K}$, доля аморфного состояния достигает максимума при $1193-1198 \mathrm{~K}$, и исчезает при $1208 \mathrm{~K}$ по мере перехода ОЦК-структуры в ГЦК. По мнению авторов [7] „наблюдаемые эффекты связаны с образованием в ходе перехода паракристаллических кластеров малых размеров с большой дисперсией параметров ячейки“. Как было показано в [34,35], и наноструктурирование [34], и аморфизация [35] кристалла приводят к одинаковым изменениям его свойств. Эти процессы увеличивают коэффициент теплового расширения $\alpha_{p}$, молярную теплоемкость $C_{p}$, коэффициент Пуассона $\mu_{p}$, и удельную энтропию $s$. Эти же процессы уменьшают модуль упругости $B_{T}$, температуру Дебая $\Theta$ и удельную поверхностную энергию $\sigma$ железа. Исходя из этого, легко понять, что появление наноструктурированной или аморфной фазы может вносить изменения в скачки параметров, которые были рассчитаны здесь для „чистого“ $\alpha \rightarrow \gamma$-перехода, и которые представлены в табл. 1. Поэтому представленные здесь результаты расчета можно использовать, как для изучения $\alpha \rightarrow \gamma$ перехода, так и для оценки вклада магнитной, наноструктурной, вакансионной либо других подсистем в свойства железа при этом переходе.

В заключение стоит отметить, что используемая в работе модель, является изотропной, что легко видеть из формулы (4). Вместе с тем, ОЦК- и ГЦК-кристаллы имеют кубическую анизотропию. Согласно [36, стр. 286] фактор упругой анизотропии, определяющий меру изотропности кристалла, для кубической структуры определяется соотношением: $A=2 C_{44} /\left(C_{11}-C_{12}\right)$, где $C_{i j}-$ упругие константы кристалла. Для упругоизотропного тела $A=1$. Если использовать для $C_{i j}$ экспериментальные данные из работы [9], то для фактора упругой анизотропии получим

для ОЦК-Fе (при $4.2 \mathrm{~K}: C_{11}=243.1, C_{12}=138.1$ и $\left.C_{44}=121.9 \mathrm{GPa}\right): A=2.322$,

для ГЦК-Fе (при $1428 \mathrm{~K}: C_{11}=154, C_{12}=122$ и $\left.C_{44}=77 \mathrm{GPa}\right): A=4.813$.

Поэтому при изучении $\alpha \rightarrow \gamma$-перехода необходимо учитывать также и изменение анизотропии кристалла железа. Но эта задача выходит за рамки настоящей работы. 


\section{5. Заключение}

Путем подгонки под значение скачка объема при $\alpha \rightarrow \gamma$-переходе в железе при $P=0$ и $T_{f}=1184 \mathrm{~K}$ определены параметры парного межатомного потенциала Ми-Леннард-Джонса для ГЦК- $\gamma$-Fe.

На основе полученных параметров в рамках единого метода рассчитаны 23 свойства $\alpha$ - и $\gamma$-фаз железа при $P=0$ и $T_{f}=1184 \mathrm{~K}$. Расчеты показали хорошее согласие с экспериментальными оценками для $\Delta V, \Delta s / k_{B}$ и $\partial T_{f} / \partial P$. Это позволяет утверждать корректность и других рассчитанных параметров, которые измеряются с большой погрешностью.

Расчеты показали, что такие свойства как: $\gamma, z, B^{\prime}(P)$, $\alpha_{p}, C_{v}$ и $C_{p}$ практически не изменяются при $\alpha \rightarrow \gamma$ переходе. Функции $B_{T}, \alpha_{p} \cdot B_{T}, s, \alpha_{p}^{\prime}(P), C_{v}^{\prime}(P), C_{p}^{\prime}(P)$, $\sigma(100), \mu_{p}, \sigma^{\prime}(T)_{\nu}, \sigma^{\prime}(T)_{P}$ и $\sigma^{\prime}(P)_{T}$ изменяются так же, как и молярный объем кристалла, т.е. в пределах $1 \%$. Параметры $\Theta, q, \Theta^{\prime}(P)$ и $X_{s c}=\sigma(100) /\left(c \cdot B_{T}\right)$ уменьшаются при $\alpha \rightarrow \gamma$-переходе так же, как увеличивается расстояние между центрами ближайших атомов $(c)$, т. е. в пределах $2-3 \%$.

На основе анализа известных из литературы экспериментальных данных показано, что даже сравнительно точно измеримые параметры как $\alpha_{p}$ и $B_{T}$ измеряются с погрешностью превышающей значения скачков этих параметров при $\alpha \rightarrow \gamma$-переходе.

Указано, что обнаруженное в работе [7] явление аморфизации (либо наноструктурирования) определенной доли железа в процессе $\alpha \rightarrow \gamma$-перехода может вносить свой вклад в изменения свойств железа при данном фазовом переходе.

\section{Благодарности}

Автор выражает благодарность С.П. Крамынину, Н.Ш. Газановой, 3.М. Сурхаевой и М.М. Гаджиевой за плодотворные дискуссии и помощь в работе.

\section{Финансирование работы}

Работа выполнена при финансовой поддержке РФФИ (грант № 18-29-11013_мк) и Программы Президиума РАН (программа № 6, грант 2-13).

\section{Конфликт интересов}

Автор заявляет, что у него нет конфликта интересов.

\section{Список литературы}

[1] L.J. Swartzendruber. Bull. Alloy Phase Diagrams 3, 2, 161 (1982).

[2] С.И. Новикова. Тепловое расширение твердых тел. Наука, M. (1974). 294 c.

[3] J.M. Besson, M. Nico. J. Geophys. Res. 95, B13, 21717 (1990).
[4] Y.C. Liu, F. Sommer, E.J. Mittemeijer. Thermochim. Acta 413, $1-2,215$ (2004).

[5] S. Klotz, Y. Le Godec, T. Strässle, U. Stuhr, Appl. Phys. Lett. 93, 9, 091904 (2008).

[6] P.I. Dorogokupets, T.S. Sokolova, K.D. Litasov. Geodynam. Tectonophys. 5, 1033 (2014).

[7] А.М. Балагуров, И.А. Бобриков, И.С. Головин. Письма в ЖЭТФ 107, 9, 583 (2018).[A.M. Balagurov, I.A. Bobrikov, I.S. Golovin. JETP Lett. 107, 9, 558 (2018)].

[8] Z. Dong, W. Li, D. Chen, S. Schónecker, M. Long, L. Vitos. Phys. Rev. B 95, 5, 054426 (2017).

[9] J. Meiser, H.M. Urbassek. Mod. Simul. Mater. Sci. Eng. 28, 5, 055011 (2020).

[10] М.Н. Магомедов. ЖТФ 85, 11, 48 (2015). [M.N. Magomedov. Tech. Phys. 60, 11, 1619 (2015).]

[11] М.Н. Магомедов. ЖТФ 87, 4, 549 (2017). [M.N. Magomedov. Tech. Phys. 62, 4, 569 (2017).]

[12] М.Н. Магомедов. Рос. нанотехнологии 14, 1-2, 19 (2019). [M.N. Magomedov, Nanotechnol. Rus. 14, 1-2, 21 (2019).]

[13] М.Н. Магомедов. ФТТ 62, 7, 998 (2020). [M.N. Magomedov. Phys. Solid State 62, 7, 1126 (2020)].

[14] М.Н. Магомедов. ФТТ 60, 5, 970 (2018). [M.N. Magomedov. Phys. Solid State 60, 5, 981 (2018)].

[15] Л. Жирифалько. Статистическая физика твердого тела, Мир, М. (1975), 383 c. [L.A. Girifalco. Statistical Physics of Materials. J. Wiley and Sons Ltd., N. Y. (1973).]

[16] Е.Ф. Пичугин. Изв. вузов. Физика 6, 77 (1962).

[17] М.Н. Магомедов.ФТТ 59, 6, 1065 (2017). [M.N. Magomedov. Phys. Solid State 59, 6, 1085 (2017).]

[18] S.S. Batsanov, A.S. Batsanov. Introduction to structural chemistry. Springer Science \& Business Media, Heidelberg (2012). 545 p.

[19] T. Horiuchi, H. Uzawa, M. Igarashi, F. Abe, T. Mohri. Calphad 26, 1, 3 (2002).

[20] J. Zhang, F. Guyot. Phys. Chem. Minerals 26, 6, 419 (1999).

[21] M. Abuin, Z. Turgut, N. Aronhime, V. Keylin, A. Leary, V. Degeorge, J. Horwath, S.L. Semiatin, D.E. Laughlin, M.E. Mchenry. Determination of Pressure Effects on the $\alpha \rightarrow \gamma$ Phase Transition and Size of Fe in Nd-Fe-B Spring Exchange Magnets (2015).

[22] N.N. Sirota, N.A. Konoplin, T.M. Soshnina. Dokl. Phys. 50, 11, 553 (2005).

[23] P.I. Dorogokupets, A.M. Dymshits, K.D. Litasov, T.S. Sokolova. Sci. Rep. 7, 41863, 1 (2017).

[24] Z.S. Basinski, W. Hume-Rothery, A.L. Sutton. Proc. Royal Soc. London. A 229, 1179, 459 (1955).

[25] M. Acet, H. Zahres, E.F. Wassermann, W. Pepperhoff. Phys. Rev. B 49, 9, 6012 (1994).

[26] V.K. Kumikov, Kh.B. Khokonov. J. Appl. Phys. 54, 3, 1346 (1983).

[27] B.B. Alchagirov, T.M. Taova, Kh.B. Khokonov. Transact. JWRI. Spec. Issue (Jpn) 30, 287 (2001).

[28] E.J. Song, H. Bhadeshia, D.W. Suh. Corrosion Sci. 77, 379 (2013).

[29] H. Chamati, N.I. Papanicolaou, Y. Mishin, D.A. Papaconstantopoulos. Surf. Sci. 600, 9, 1793 (2006).

[30] D.J. Dever, J. Appl. Phys. 43, 8, 3293 (1972). 
[31] В.Е. Зиновьев, Теплофизические свойства металлов при высоких температурах. Справочник. Металлургия, М. (1989), $384 \mathrm{c}$.

[32] D. Antonangeli, G. Morard, N.C. Schmerr, T. Komabayashi, M. Krisch, G. Fiquet, Y. Fei. Proc. Nat. Academy Sci. 112, 13, 3916 (2015).

[33] Y. Nishihara, Y. Nakajima, A. Akashi, N. Tsujino, E. Takahashi, K.I. Funakoshi, Y. Higo. Am. Mineral. 97, 8-9, 1417 (2012).

[34] М.Н. Магомедов. Рос. нанотехнологии 10, 1-2, 76 (2015). [M.N. Magomedov. Nanotechnolog. Rus. 10, 1-2, 89 (2015).]

[35] М.Н. Магомедов. ЖТФ 90, 10, 1731 (2020). [M.N. Magomedov. Tech. Phys. 65, 10, 1659 (2020).]

[36] М.П. Шаскольская. Кристаллография. Высш. шк., М. (1976). $391 \mathrm{c}$.

Редактор К.В. Емцев 